\title{
Explicit Computational Investigations on the Effect of Microstructure in Solid Oxide Fuel Cell Electrodes
}

\author{
David S. Mebane, Arun M. Gokhale and Meilin Liu \\ School of Materials Science and Engineering, Georgia Institute of Technology \\ 771 Ferst Dr. NW, Atlanta, GA, 30332-0245
}

It is well known in the field of solid oxide fuel cells (SOFCs) that interfacial resistance in the electrodes causes the majority of power losses in the cell. These interfacial losses, in turn, intricately depend on the microstructure of the electrodes. In a porous composite (two solid phase) cathode, microstructural features that influence the overall resistance include the triple-phase boundary (onedimensional) between an ionically conducting phase (electrolyte), mixed ionic-electronic conducting phase (MIEC) and the gas, two-phase areas, grain boundaries, and pores. The dependence of performance on microstructure arises from these features' ability to hinder or facilitate the transport of electrons (or electron holes), oxygen ions (or oxygen vacancies) and gaseous species, as well as chemical or electrochemical reactions between these species (see Figure 1 for an illustration). Deconvoluting the effect of microstructure on electrode performance becomes even more complicated when one considers functionally graded electrodes, wherein the microstructure changes dramatically throughout the thickness of the electrode, from the current collector to the electrolyte (see Figure 2).

Our research efforts in this area will combine novel microstructural characterization and modeling techniques with electrochemical modeling and testing to construct microstructurally and electrochemically sensitive computational models. Existing electrochemical models for SOFCs typically rely on either effective or averaged microstructural parameters such as porosity, tortuosity and internal surface area [1,2] or investigate simplified geometries [3,4]. While such studies are certainly valuable, their inherent approximations with respect to the microstructure leave structureproperty relationships in fuel cells poorly defined, and precise models for the purpose of rational materials design are still lacking. Into this picture enters state-of-the-art techniques in microstructural characterization and simulation. Serial-sectioning methods of microstructural reconstruction (see Figure 3a), and advanced microstructural modeling techniques (Figure 3b) allow for accurate, digitized representations of complex materials. Equations governing transport, chemical and electrochemical reaction rates, and electrical states can be applied throughout this digitized space, which may then be analyzed through finite element analysis.

This technique has the potential to serve as either an advanced characterization tool or an optimization tool. The reconstructions may be compared with experiments in order to validate the model or provide insight into unknown properties of the electrode such as specific reaction rate constants. The microstructural simulations can explicitly show the effects of microstructural changes on electrochemical response, and can be used as tools for design optimization [5].

References

[1] S. B. Adler et al., J. Electrochem. Soc., 143 (1996) 3554.

[2] P. Costamagna et al., Electrochim. Acta 43 (1998) 375.

[3] J. Fleig and J. Maier, J. Eur. Ceram. Soc. 24 (2004) 1343. 
[4] C. W. Tanner et al., J. Electrochem. Soc. 144 (1997) 21.

[5] This work was supported by DoE-NETL SECA Core Technology Program (Grant No. DEFC26-02NT41572).

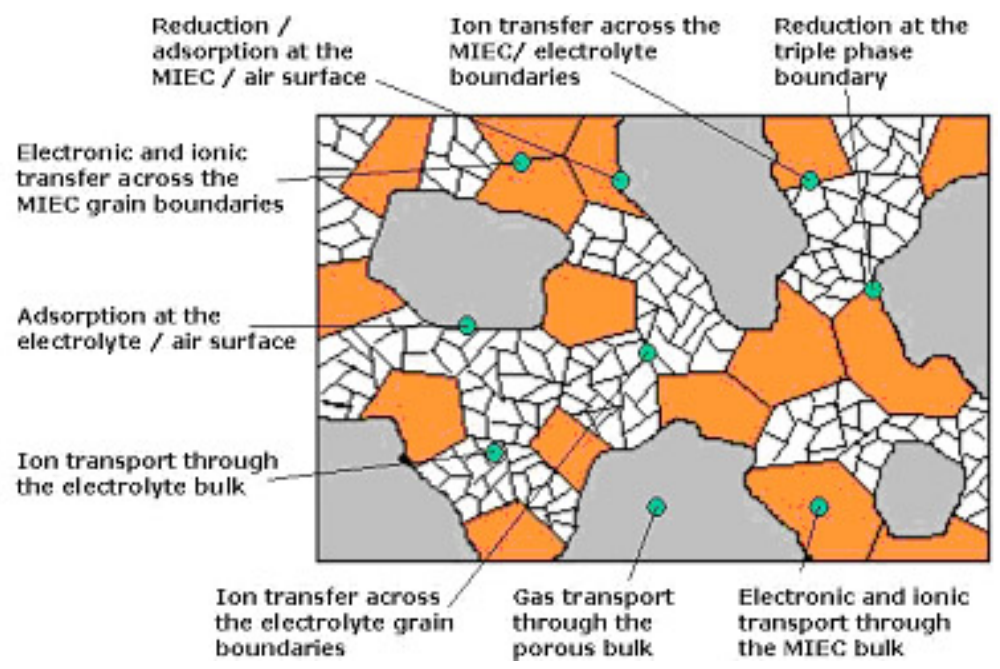

FIG. 1. Schematic of a cathode section, indicating the influence of microstructure on properties.

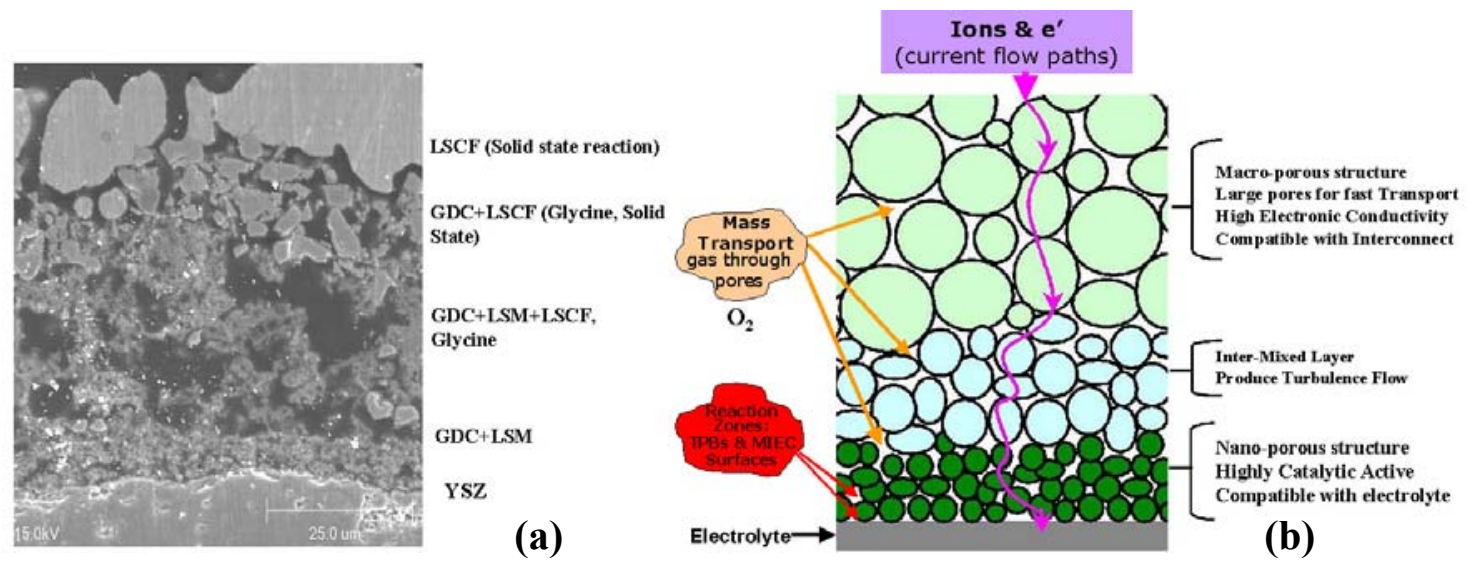

FIG. 2. (a) SEM image and (b) schematic of a functionally graded SOFC cathode (S. Zha).
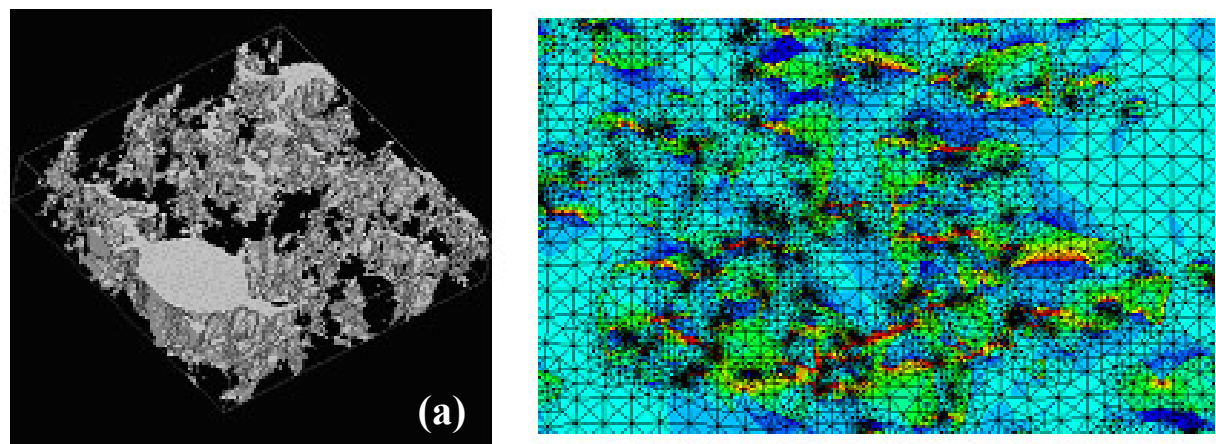

(b)

FIG. 3. (a) Reconstruction of porosity in a Mg alloy (S.-Y. Lee) and (b) finite element meshing of a 2-D simulated microstructure (A. Sreeraganathan). 